\title{
Les études du handicap en géographie. Fondements, état des lieux et ouvertures
}

\section{Jason Borioli, Lausanne}

\section{Introduction}

Il fait froid en cette fin de mois d'octobre. Étienne marche dans une rue en bord de route. Comme tous les jeudis, il y a le marché aux puces, mais il est encore tôt, la voie est dégagée. Il peut donc marcher où il aime, sur les pavés derrière les abribus, plutôt qu'en bord de route, où il n'apprécie guère le bruit de la circulation et où il a l'impression de déranger les personnes qui attendent leur transport. Plus loin, après une montée de quelques mètres, il rejoint une ruelle encastrée entre deux rangées de bâtiments. C'est calme, il peut éviter la rigole qui sépare la rue en deux sur toute sa longueur et où il a failli se tordre une cheville voici quelques mois. Il arrive au feu. Il est au rouge, mais le bus est très loin, il y va quand même. Il aime cette rue, cette vallée entre deux rangées d'immeubles, où il a l'impression d'être sur des rails et en même temps de se noyer entre les bâtiments, cette évidence visuelle qui lui indique tout naturellement par où passer, lui laissant le loisir de marcher sans réfléchir, sous le mode de la flânerie.

Il fait froid en cette fin de mois d'octobre. Anne se déplace en fauteuil roulant dans une rue en bord de route. Comme tous les jeudis, il y a le marché aux puces, mais il est encore tôt, la voie est dégagée. Elle passe néanmoins de l'autre côté, en bord de route, pour éviter les pavés - et puis la proximité des gens qui attendent leur bus lui fait plaisir. Le trottoir est large et à cette heure-ci il n'y a plus trop de monde. Plus loin, la route est barrée en raison de travaux là où elle avait prévu de passer. Elle doit passer par la montée et rejoindre la ruelle un peu plus haut, mais la pente est trop raide pour la franchir sans aide. Elle bloque sa chaise, regarde autour d'elle et demande au monsieur qui marche dans sa direction de l'accompagner. Il l'accompagne et lui demande si elle souhaite qu'il l'accompagne ailleurs. Elle décline et remercie. Elle est sur du plat, ça va aller. Plus loin, elle est attirée par un produit dans la vitrine de l'autre côté de la ruelle, mais elle va devoir négocier la rigole. Enfin, tant pis, de toute façon les vingt centimètres de marche à l'entrée du magasin rendent l'accès rédhibitoire et cette fois-ci elle n'a pas envie de demander de l'aide. Elle arrive au feu rouge et attend qu'il passe au vert. Elle déteste ce passage car il est en pente. Elle doit à chaque fois lutter pour aller droit et négocier le petit seuil en dévers n'est franchement pas drôle. L'autre côté de la rue est pavé, mais elle n'a pas le choix.

Il fait froid en cette fin de mois d'octobre. Chantal marche en balayant avec sa canne dans une rue en bord de route. Comme tous les jeudis, il y a le marché aux puces, elle entend des gens en train de monter des stands. Elle cherche de ses oreilles l'arrêt du bus et elle trouve le bord du trottoir. Elle préfère passer par là, même s'il y a des gens qui montent et descendent des bus, parce qu'il est facile de suivre le trottoir et parce que le bruit de la circulation est un bon repère pour elle. De plus, quand elle ne sent plus la présence du mur de l'abribus et du kiosque attenant, elle sait qu'elle peut tourner à gauche pour monter vers la ruelle. C'est mieux que l'allée derrière les abribus, où sa canne se bloque entre les pavés et où, les jours de marché, il faut faire attention aux livreurs qui laissent ouvertes les portes arrière de leur camionnette. Elle sent que ça commence à monter, elle sait alors qu'elle est dans la bonne direction. Elle marche lentement et sonde l'espace avec sa canne, car il y a beaucoup d'arbres et de poteaux. Elle attend le bruit d'une fontaine sur sa gauche, car c'est à hauteur de cette fontaine qu'elle fera un quart de tour à droite pour s'engager dans la ruelle. Elle espère que la fontaine coule encore malgré le froid. Elle l'entend et peut donc entrer dans la ruelle. Ici, ce qu'elle trouve très pratique, c'est la rigole. C'est un excellent repère pour avancer, il suffit de la suivre avec sa canne pour ne pas dévier. La rue regorge d'odeurs - la boulangerie, la boucherie et la chaleur et le souffle des entrées des grands magasins. Chantal entend un bus passer devant elle. Elle sait ainsi qu'elle approche des feux. Elle craint cet endroit, car la rigole se termine quelques pas avant le passage pour piétons et elle ne trouve jamais le poteau de signalisation. Ce poteau est difficile à repérer, car il n'est pas dans l'axe et une fois sur deux elle croit l'avoir trouvé alors que c'est une poubelle qui se trouve à proximité. Souvent elle doit donc se fier à la circulation.

Cette introduction, volontairement hétérodoxe à des fins d'illustration, met en scène trois fragments de vie urbaine et constitue une synthèse de parcours commentés, dirigés par l'auteur entre septembre 2008 et septembre 2009. Ces parcours sont recomposés, certes, mais bien réels et rendent compte d'expériences quotidiennes cristallisées en un décalage entre la structure de nos sociétés et les besoins d'une partie de la population, en l'occurrence les «personnes handicapées» (la terminologie est explicitée plus loin dans le texte). Ce 
décalage a été décrit et commenté du point de vue descriptif (Borioli \& LAUB 2007b; IMRIE 1996a; Kitchin et al. 1998; Matthews \& Vujakovic 1995) et conceptuel (Fougeyrollas et al. 1998; Fougeyrollas \& Noreau 2007; Organisation Mondiale de la Santé 1988 , 2001). Il renvoie notamment à la théorie gibsonienne de l'affordance et à son concept de niche (GIBSON 1977, 1986[1979]), une affordance étant les possibilités d'interaction entre un être vivant et les éléments de son environnement et une niche étant un ensemble d'affordances. C'est ce décalage, ces «situations de handicap», que cet article se propose de passer en revue.

Au niveau des sciences sociales, un consensus s'est formé ces dernières années autour de l'explication de ces «situations de handicap». Schématiquement, à un désavantage intrinsèque à la personne - la déficience (organique), l'incapacité (fonctionnelle) - se greffe un désavantage extrinsèque qui est le résultat de la structure de l'environnement dans lequel elle évolue. Celle-ci est donc la victime d'une «violence structurelle» (BoRIOLI, LAUB \& Racine 2005), le résultat de ce que ImRIE (1996a) appelle design apartheid, c'est-àdire la (re)production d'environnements produits par et pour une «norme» fonctionnelle, «par des humains aux corps intacts pour des humains aux corps intacts» (MurPhy 1993[1987]: 190). Selon cette perspective, la «personne handicapée» est la victime d’une «ségrégation fonctionnelle», au double sens du rapport à des aptitudes fonctionnelles et à des logiques sous-jacentes qui ne sont pas étrangères à d'autres formes de ségrégation, comme le montre la comparaison entre l'inaccessibilité urbaine et la politique ségrégationniste du Jim Crow aux Etats-Unis (BoRIOLI \& LAUB 2007b: 160 164). L'environnement, dans toutes ses dimensions, ne saurait ainsi être réduit ni à l'épaisseur et à l'«inertie» (IMRIE 1996a) de son héritage historique, ni à une simple mise en forme de normes sociales.

On retrouve ici une conception lefebvrienne, en cela que l'espace est à la fois un produit social et un producteur de vie sociale (LeFebvre 1974). D'emblée, la «situation de handicap» mobilise un savoir (aussi) géographique, ne serait-ce que parce qu'elle renvoie à des formes et processus spatiaux (BRUNET 1990: 31 45; Matthey 2008: 22-25). Cependant, la géographie ne s'est guère intéressée au handicap. Les travaux portant sur la question sont relativement récents (BUTLER 1994; Butler \& Bowlby 1997; Butler \& Parr 1999; GleEson 1996, 1999a; GolledGe 1993, 1994, 1996; HAHN 1986; ImRIE 1996a, 1996b; KтTCHIN et al. 1997, 1998; MatThews \& Vujakovic 1995; PARK, RadFoRd \& VICKERS 1998) et n'ont pas intégré à leur réflexion l'ensemble des principaux concepts et théories des études du handicap. Il convient dès lors, dans le cadre d'une revue de ces travaux en géographie, d'interroger l'évolution des modèles et théories du handicap.

\section{Les études des situations de handicap}

Les Disability Studies (DAvIS 1997), expression traduite dans cet article par «études des situations de handicap», émergent en tant que discours cohérent dans les pays anglo-saxons à partir du milieu du $\mathrm{XX}^{\mathrm{e}}$ siècle, faisant fond sur des organisations de droits civiques et de vie autonome (Albrecht, Ravaud \& Stiker 2001; GleEson 1999a: 16-33; Ravaud 2001). Historiquement, ces études ont orienté leurs efforts davantage vers la praxis que vers la théorisation, dans une logique militante et en réaction à une conception du handicap perçue comme réductrice et par trop médicale (GLEESON 1999a: 16-18; Oliver 1990, 1996). Il s'agissait dès lors d'affranchir les «personnes handicapées» de l'oppression dont elles étaient les victimes. En revanche, un autre courant, davantage représenté dans le contexte francophone (Blanc 2006; STIKer 2005), paraît plus concerné par des efforts de théorisation.

\subsection{Des définitions du handicap}

Le mot «handicap» est récent et a remplacé «infirmité», «invalidité» et «débilité» (BorIOLI \& LAUB 2007a: 2545). Pour le sens commun, il revêt plusieurs significations. Il se rapporte à une pathologie biologique (par exemple une sclérose en plaques), à une déficience fonctionnelle (une paraplégie), aux conséquences de cette pathologie ou déficience sur les capacités de la personne (une incapacité à la marche), ou à une participation sociale limitée, voire à toutes ces dimensions à la fois.

Du point de vue conceptuel, trois modèles du handicap ont été proposés (Borioli \& LAUB 2007a; Ravaud 1999). Premièrement, un modèle individuel ou biomédical qui explique en termes de déficience le désavantage social d'une personne dite «handicapée», en faisant abstraction du contexte (OrGANISAtion Mondiale de la Santé 1988: 23-28). C'est ainsi que la participation sociale limitée d'une «personne handicapée» s'explique par ses propres manques. Le modèle social, développé par des personnes concernées en réaction au modèle biomédical, explique ce désavantage social par le manque de prise en compte des besoins d'une partie de la population, celle qui se déplace, s'exprime ou perçoit différemment de la plupart des gens (Oliver 1990, 1996). Le «handicap» devient une pathologie avant tout sociale. Un troisième modèle, qualifié de systémique (FougEYrollas \& NOREAU 2007) ou environnementaliste (BLANC 2006) est cristallisé dans le modèle conceptuel du «Processus de Production du Handicap» (PPH, voir fig. 1) (FouGEYROLLAS et al. 1998) et, dans une moindre mesure (Stiker 2002, 2005), dans la Classification internationale du fonctionnement, du handicap et de la santé (CIF) (Organisation Mondiale de la Santé 2001). Le modèle systémique définit le handicap comme le 


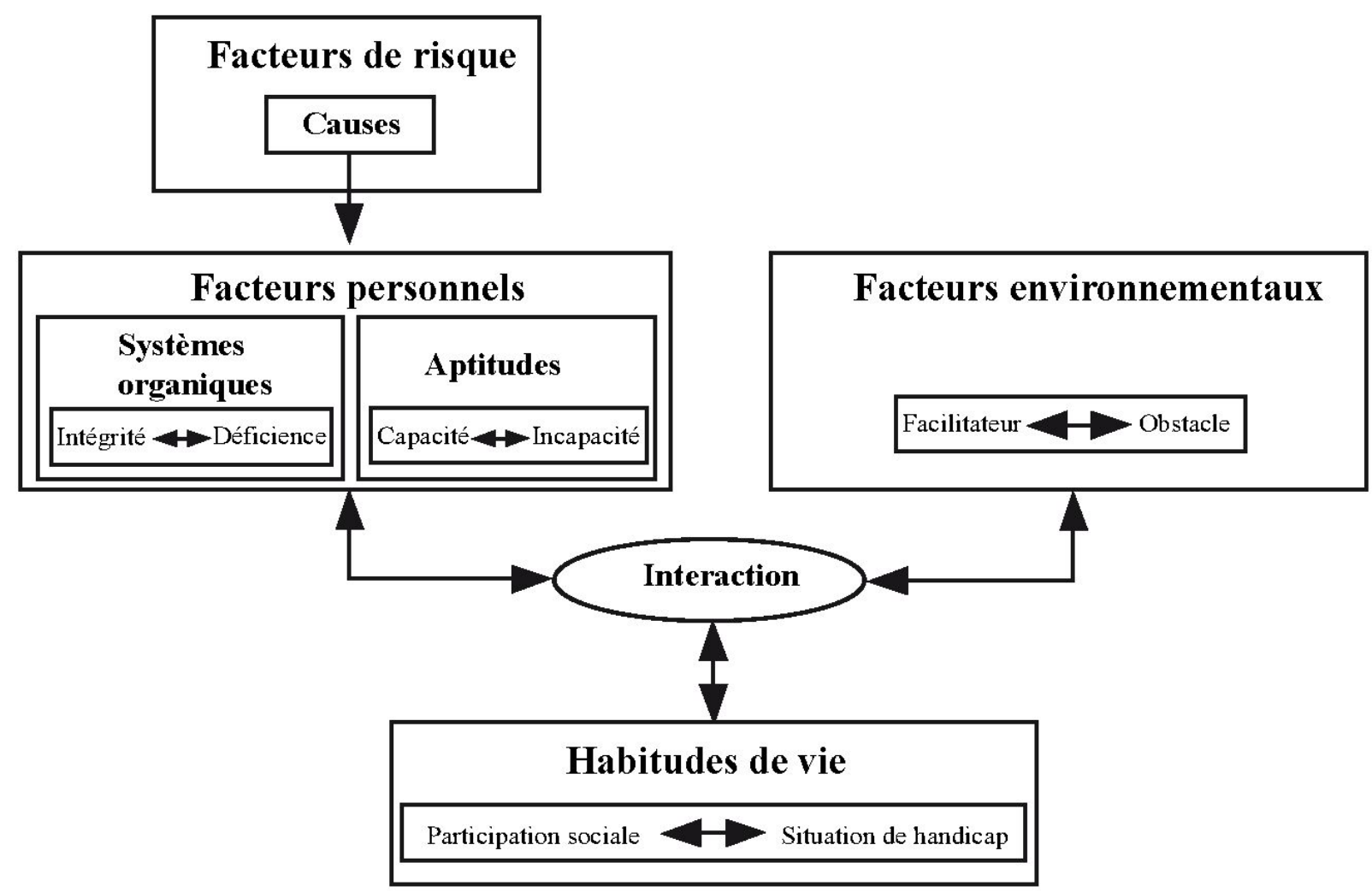

Fig. 1: Processus de Production du Handicap (PPH). Modèle explicatif des causes et conséquences des maladies, traumatismes et autres atteintes à l'intégrité ou au développement de la personne.

Disability Creation Process (DCP). An explanatory model of the causes and consequences of disease, trauma and disruptions to a person's integrity of development.

Prozess der Produktion des Handicaps. Erklärungsmodell der Ursachen und Konsequenzen von Krankheiten, Traumata und anderen Beeinträchtigungen der Integrität oder Entwicklung einer Person.

Source: FougeYRoLLAS et al. 1998

résultat de la rencontre entre une personne ayant des incapacités et un environnement plus ou moins adapté à ses besoins. Aussi, la notion situationnelle du handicap, qui est antérieure aux PPH et à la CIF, trouve une assise formelle et prend corps dans l'expression «situation de handicap» (Borioli \& LaUb 2007a).

Il convient sur ces bases de préciser quelques notions sémantiques. Une attention particulière a en effet été consacrée dans le cadre des études du handicap au rôle des mots, conçus comme des entités à même de produire du contexte et donc d'induire une certaine réalité (BoRIOLi \& LAUB 2007a). Ainsi, en référence à la conception systémique, l'expression «personne en situation de handicap» tend désormais à remplacer celle de «personne handicapée». L'expression utilisée dans cet article est en revanche celle de «personne ayant des incapacités», en référence explicite au PPH, en cela que la personne ayant des incapacités peut vivre «de façon variable des situations de handicap selon la qualité du contexte de réalisation des habitudes de vie de leur choix» (Fougeyrollas \& Noreau 2007: 58). Le cadre anglophone se caractérise également par un manque de consensus, notamment autour des expressions «people with disabilities» et «disabled people», les tenants de la première affirmant rendre leur dignité de personne aux personnes concernées, les partisans de la deuxième mettant l'accent sur l'expérience du handicap comme forme d'oppression sociale (Gleeson 1999a: 9; Oliver 1990: xiii).

\subsection{Les théories du handicap}

Les études des situations de handicap ont également produit des théories visant à expliquer le fait que, historiquement, les sociétés n'ont pas su intégrer pleinement l'infirmité. Quatre grandes théories ont été proposées à ce jour, mobilisant des concepts et données sociologiques, anthropologiques et psychanalytiques: la théorie de la déviance et du stigmate, la théorie de l'oppression sociale, la théorie culturaliste et la théorie 
de la liminalité, cette dernière ayant été complétée par une explication basée sur le concept de Double (pour une revue exhaustive, voir STIKER 2005: 191-226).

La théorie de la déviance et du stigmate (BECKER 1985 [1963]; Davis 1961; Freidson 1965; Goffman 1975 [1963]) décrit le processus social qui conduit au rejet des personnes ayant des incapacités. Ce processus est expliqué par les caractéristiques des personnes jugées déviantes:

«un individu qui aurait pu aisément se faire admettre dans le cercle des rapports sociaux ordinaires possède une caractéristique telle qu'elle peut s'imposer à l'attention de ceux d'entre nous qui le rencontrons, et nous détourner de lui, détruisant ainsi les droits qu'il a vis-à-vis de nous du fait de ses autres attributs» (GoFFMAN 1975 [1963]: 15).

La théorie de l'oppression sociale (Oliver 1990,1996), qui rejoint le modèle social, conçoit le handicap en tant que problème de société, au sens où les personnes ayant des incapacités seraient les victimes d'oppression de la part d'une société capitaliste qui rejette celles et ceux qui ne peuvent pas contribuer au rythme de production dicté par l'économie de marché. Cette théorie conteste alors le caractère historique du rejet de l'infirmité et avance que ce rejet est essentiellement le produit du capitalisme (voir par exemple GLEESON 1999a: 22-24). Dans son extrême, elle fait abstraction des dimensions personnelles et a dès lors été critiquée en tant que «désincarnation» et «occultation de la corporéité» du handicap (GleEson 1999a: 19-20; Ravaud 1999: 64).

La théorie culturaliste (LANE 1984) se fonde sur les expériences des personnes sourdes et pratiquant la langue des signes, conçue comme spécificité culturelle. Les théories de l'oppression sociale et culturaliste partagent l'idée que seules les personnes ayant des incapacités peuvent se prononcer sur le handicap et que là réside l'unique possibilité de s'affranchir d'une vision biomédicale qui participe à la reproduction de l'oppression sociale.

La théorie de la liminalité (BLANC 2006, 2010; GARDou 1997, 2000, 2005; Murphy 1993 [1987]; Stiker 2005) se concentre moins sur la notion de rejet et se base sur les notions de rites de passage (VAN GENNEP 1981[1909]) et de seuil (Turner 1990[1969]). La liminalité indique en effet une situation de seuil, au sens où la personne ayant des incapacités se trouverait «dans des limbes sociaux où [elle] est maintenu[e] en dehors du système social formel» (MuRPHY 1993 [1987]:184), en référence à des situations dans lesquelles se trouvent des personnes qui traversent des rites de passage, au moment où elles ont quitté le statut d'avant le rite mais où elles n'ont pas encore atteint le statut auquel ce même rite doit leur permettre d'accéder. La différence entre ces situations et la situation des personnes ayant des inca- pacités résiderait dans le fait que ces dernières seraient dans une liminalité constante, sur le seuil, ni exclues, ni intégrées. MurPHy (1993[1987]: 184) décrit cette liminalité en ces termes:

«Les handicapés à long terme ne sont ni malades ni en bonne santé, ni morts ni pleinement vivants, ni en dehors de la société ni tout à fait à l'intérieur. (...) L'invalide (...) passe sa vie (...) dans un isolement partiel en tant qu'individu indéfini et ambigu».

STIKER (2005) bâtit sur cette théorie et montre que, en filigrane des différents systèmes de pensées et pratiques sociales à l'égard de l'infirmité, ce que nous nommons aujourd'hui «handicap» reste source d'inquiétude. Nous serions alors face à une forme «d'inquiétante étrangeté» et, possiblement, à un invariant anthropologique. De ce point de vue, l'infirmité ne peut être ni totalement accueillie, ni totalement rejetée, car il s'agit d'un Double qui nous appartient en même temps qu'il nous répugne, «à la manière d'une ombre troublante et dangereuse dont il faudrait se protéger sans cesser de faire un avec elle» (STIKer 2005: 216) et «pas tout à fait dehors, pas tout à fait dedans, dans les seuils ou aux frontières» (ibid. 218). Le syndrome bien connu du NIMBY (Not In My Backyard) (GLEESON 1999a: 156-160) trouve ici une explication anthropologique et psychanalytique.

\section{Les études des situations de handicap en géographie}

Les premières études du handicap en géographie, issues de la géographie médicale, datent de la fin des années 1970. Elles portaient sur la cartographie de différentes incapacités et sur les capacités de cognition spatiale des personnes ayant des incapacités mentales (GolledGE, PARNICKY \& RAYNER 1979; Golledge et al. 1991) ou sensorielles (KITCHIN, BladES \& GolledGe 1997; KITCHIN et al. 1998), selon une conception plutôt biomédicale du handicap (PARK, RADFord \& VicKers 1998; Philo 2009). Un autre courant, revendiquant une posture post-positiviste et se référant explicitement à la notion de droits humains, a porté son attention dès les années 1990 sur la composante sociale du handicap, au sens d'obstacles physiques et sociaux qui empêchent une pleine participation sociale des personnes concernées (GLEESON 1996, 1999a; IMRIE 1996a). Un troisième courant, qui émerge également depuis les années 1990, tâche de rendre compte des interactions entre les dimensions individuelles et sociales du handicap (BUTLER \& Bowlby 1997; Butler \& Parr 1999; Gleeson 1999a).

En ce qui concerne la terminologie, les débats internes aux études du handicap ont été reproduits en géographie (GleEson 1999a; Golledge 1996; IMrIe 1996a; PARR 1997). Ce reflet a été cristallisé dans les débats opposant les problématiques comportementales et his- 
torico-matérialistes (GLEESON 1996, 1999a; GolLEDGE 1996; IMrIE 1996b). GolledGe (1993, 1996) estime ainsi qu'une approche géographique du handicap doit porter sur l'étude des compétences spatiales des personnes ayant des incapacités et cela dans un but résolument appliqué, visant la récolte de données pouvant fournir des informations utiles à l'aménagement d'espaces urbains et au développement d'aides au déplacement, notamment pour les personnes aveugles ou malvoyantes (GolledGe 1991; GolledGe et al. 1991; Golledge \& Timmermans 1990; Jacobson \& Kitchin 1995; Kitchin \& JACOBSON 1997). En revanche, GLEESON $(1996,1997,1999 a, 1999 b)$ et IMRIE (1996a), considèrent que de telles études reproduisent la conception biomédicale du handicap et négligent la perspective socio-constructiviste. Aussi, leur intérêt porte sur la socialisation des incapacités dans différents contextes historiques et géographiques (GLEEson 1999a) et, dans une perspective de géographie du pouvoir (RAFFESTIN 1980), sur les processus qui conduisent à la production d'espaces qui ne tiennent pas compte des besoins des personnes ayant des incapacités (CHouinard 1997; GLEESON 1999a) en référence notamment au concept de design universel (IMRIE 1996a).

Cette brève revue montre que les travaux géographiques sur le handicap constituent le miroir des modèles issus des Disability Studies, alors que les théories du handicap n'ont pas été intégrées au corpus des réflexions géographiques. Faut-il y voir le signe d'une «vocation purement descriptive de la géographie», pour reprendre les termes de BENOIST (2001: 228), lorsqu'il pose la question de savoir si la géographie

«ne se caractérise-t-elle tout de même pas, essentiellement, par rapport à d'autres discours alternatifs, par son régime de discursivité à la base descriptif»?

La géographie serait-elle dès lors condamnée par son sens étymologique premier à une description des situations de handicap, de leur distribution spatiale, éventuellement des contextes sociaux et matériels qui augmentent ou réduisent les situations de handicap? La prochaine et dernière partie de cette contribution questionne les potentialités théoriques que la géographie pourrait mobiliser dans le cadre des études des situations de handicap.

\section{Ouverture: quelle(s) théorie(s) pour une géographie du handicap?}

En suivant BRunet (1990:31-45) et MATTHey (2008:2225), le projet spécifique à la géographie serait celui de penser les processus sous-jacents aux formes spatiales. Dès lors, les situations de handicap sont-elles susceptibles d'être théorisées du point de vue de la géographie, comme l'anthropologie a su le faire?
En suivant les deux problématiques géographiques évoquées plus haut dans le texte-comportementale et historico-matérialiste-, la théorie des affordances et la théorie de la liminalité semblent contenir en elles des éléments susceptibles d'être exploités en géographie. D'une part, les interactions entre personnes ayant différentes incapacités et l'environnement construit peuvent être théorisées en termes d'affordances, en définissant un cadre qui permet d'étudier ce que l'environnement fournit à chaque personne en fonction de ses caractéristiques. De par son caractère écologique, le PPH s'insère également dans cette perspective (Fougeyrollas \& Noreau 2007) et les commentaires recomposés présentés en introduction donnent un exemple de données pouvant être analysées dans le cadre théorique des affordances. D'autre part, la théorie de la liminalité paraît particulièrement intéressante pour une approche géographique du handicap en cela qu'elle mobilise une réflexion territoriale fondée non seulement sur l'exclusion mais aussi sur la notion plus fine de seuil et qu'elle peut rendre compte de territorialités spécifiques aux personnes ayant des incapacités, territorialités qui ne sauraient être réduites à l'exclusion, comme cela est également illustré par les parcours commentés présentés en introduction.

Ainsi, en complément des actuels champs d'investigation, notamment la problématique de la production et de la régulation de l'espace urbain et l'analyse des modes de participation, sous-jacente à la réflexion autour du design universel, les théories de l'affordance et de la liminalité semblent fournir un terreau fertile pour la réflexion théorique des situations de handicap du point de vue géographique.

\section{Bibliographie}

Albrecht, G.L., Ravaud, J.-F. \& H.J. Stiker (2001): L'émergence des Disability Studies: état des lieux et perspectives. - In: Sciences Sociales et Santé 19: 43-73. BECKER, H.S. (1985 [1963]): Outsiders: études de sociologie de la déviance. - Paris: Métaillé

Benoist, J. (2001): En quoi la géographie peut-elle importer à la philosophie ? - In: BENOIST, J. \& F. MERLINI (éds): Historicité et spatialité. Le problème de l'espace dans la pensée contemporaine. - Paris: Librairie philosophique J. Vrin: 221-247.

Blanc, A. (2006): Le handicap ou le désordre des apparences. - Paris: Armand Colin.

Blanc, A. (2010): Handicap et liminalité: un modèle analy tique. - In: Alter - Revue européenne de recherche sur le handicap 4: 38-47.

BoRIOLI, J., LAUB, R. \& J.-B.Racine (2005): Marginalization of wheelchair-bound people as violence. In search of supportive urban environments. - In: Murayama, Y. \& Q. Du (éds): Cities in global perspective. Diversity and transition. - Tokyo: College of Tourism, Rikkyo 
University, with International Geographical Union, Urban Commission: 350-363.

Borioli, J. \& R. LAUB (2007a): Des acceptions du handicap. L'évolution du mot «handicap» de ses origines à nos jours. - In: Borioli, J. \& R. LAUB (éds): Handicap: de la différence à la singularité. Enjeux au quotidien. - Genève: Médecine \& Hygiène: 25-45.

BORIOLI, J. \& R. LAUB (2007b): La ville en fauteuil roulant. Expériences vécues par les personnes concernées. - In: Borioli, J. \& R. LaUb (éds): Handicap: de la différence à la singularité. Enjeux au quotidien. - Genève: Médecine \& Hygiène: 155-185.

BRUNET, R. (1990): Espaces produits. - In: BRUNET, R. \& O. Dollfus (éds): Géographie universelle. Mondes nouveaux. - Paris: Hachette, Montpellier: Reclus: 3145.

BUtLER, R.E. (1994): Geography and vision-impaired and blind populations. - In: Transactions of the Institute of British Geographers 19:366-368.

BUtLER, R.E. \& S. Bowlby (1997): Bodies and spaces: an exploration of disabled people's experiences of public space. - In: Environment and Planning D: Society and Space 15: 411-433.

Butler, R.E. \& H. PArr (éds) (1999): Mind and body spaces. Geographies of illness, impairment and disability. - London: Routledge.

Chouinard, V. (1997): Making space for disabling differences: challenging ableist geographies. - In:Environment and Planning D: Society and Space 15: 379-387.

DAvIS, F. (1961): Deviance disavowal: the management of strained interaction by the visibly handicapped. - In: Social Problems 9: 120-132.

DAvis, L.J. (1997): The disability studies reader. - New York, London: Routledge.

Fougeyrollas, P., Cloutier R., Bergeron, H., Côté, J. \& G. ST-Michel (1998): Classification québécoise. Processus de production du handicap. - Québec: Réseau International sur le Processus de Production du Handicap.

Fougeyrollas, P. \& L. Noreau (2007): L'environnement physique et social. Une composante conceptuelle essentielle à la compréhension du processus de production du handicap. L'exemple des personnes ayant une lésion médullaire. - In: BorioLi, J. \& R. LAub (éds): Handicap: de la différence à la singularité. Enjeux au quotidien. - Genève: Médecine \& Hygiène: 47-69.

Freidson, E. (1965): Disability as social deviance. - In: Sussman, M.B. (éd.): Sociology and rehabilitation. - Washington, D.C.: American Sociological Association: 71-99.

Gardou, C. (1997): Les personnes handicapées exilées sur le seuil. - In: Revue européenne du handicap mental 4: 6-17.

Gardou, C. (2000): Handicap, conformité et situation de seuil. - In: Prévenir 39: 71-82.

Gardou, C. (2005): Fragments sur le handicap et la vulnérabilité. - Ramonville Sainte-Agnès: Érès.
Gibson, J.J. (1977): The theory of affordances. - In: Shaw, R.E. \& J. BRANsford (éds): Perceiving, acting and knowing. - Hillsdale: Lawrence Erlbaum: 62-82.

Gibson, J.J. (1986 [1979]): The ecological approach to visual perception. - Hillsdale: Lawrence Erlbaum.

GleEson, B.J. (1996): A geography for disabled people? - In: Transactions of the Institute of British Geographers 21: 387-396.

GleEson, B.J. (1997): Disability studies: a historical materialist view. - In: Disability \& Society 12: 179-202. Gleeson, B.J. (1999a): Geographies of disability. London, New York: Routledge.

GleEson, B.J. (1999b): Can technology overcome the disabling city? - In: Butler, R.E. \& H. PARR (éds): Mind and body spaces: geographies of illness, impairment and disability. - London: Routledge: 98-118.

GoffMan, E. (1975 [1963]): Stigmate:les usages sociaux des handicaps. - Paris: Minuit.

GolledGe, R.G. (1991): Tactual strip maps as navigational aids. - In: Journal of Visual Impairment and Blindness 85: 296-301.

GolLEDGE, R.G. (1993): Geography and the disabled: a survey with special reference to vision impaired and blind populations. - In: Transactions of the Institute of British Geographers 18: 63-85.

Golledge, R.G. (1994): A response to Ruth Butler. - In: Transactions of the Institute of British Geographers 19:369-372.

Golledge, R.G. (1996): A response to Gleeson and Imrie. - In: Transactions of the Institute of British Geographers 21: 404-411.

Golledge, R.G., Loomis, J.M., Klatzy, R.L., Flury, A. \& X.L. YANG (1991): Designing a personal guidance system to aid navigation without sight: progress on the GIS component. - In: International Journal of Geographical Information Systems 5: 373-395.

Golledge, R.G., PARnicky, J.J. \& J.N. Rayner (1979): An experimental design for assessing the spatial competence of mildly retarded populations. - In: Social Science and Medicine 13D: 292-295.

Golledge, R.G. \& H. Timmermans (1990): Applications of behavioural research on spatial problems I: Cognition. - In: Progress in Human Geography 14: 57-99.

HaHn, H. (1986): Disability and the urban environment: a perspective on Los Angeles. - In: Environment and Planning D: Society and Space 4: 273-288.

ImRIe, R. (1996a): Disability and the city. International perspectives. - London: Paul Chapman.

IMRIE, R. (1996b): Ableist geographers, disablist spaces: towards a reconstruction of Golledge's «Geography and the disabled». - In:Transactions of the Institute of British Geographers 21:397-403.

JACOBSON, R.D. \& R.M. Kitchin (1995): Assessing the configurational knowledge of people with visual impairment and blindness. - In: Swansea Geographer 32: $14-24$ 
Kitchin, R.M., Blades, M. \& R.G. Golledge (1997): Understanding spatial concepts at the geographic scale without the use of vision. - In: Progress in Human Geography 21: 225-242.

KITCHIN, R.M. \& R.D. JACOBSon (1997): Techniques to collect and analyze the cognitive map knowledge of persons with visual impairment or blindness: issues of validity. - In: Journal of Visual Impairment and Blindness 91:393-400.

Kitchin, R.M., Jacobson, R.D., Golledge, R.G. \& M. BlADES (1998): Belfast without sight: exploring geographies of blindness. - Irish Geography 31: 34-46.

LANE, H. (1984): When the mind hears. A history of the deaf. - London: Random House.

LefeBvRe, H. (1974): La production de l'espace. - Paris: Anthropos.

Matthews, M.H. \& P.Vujakovic (1995): Private worlds and public spaces: mapping the environmental values of wheelchair users. - In: Environment and Planning A 27: 1069-1083.

Matthey, L. (2008): Le quotidien des systèmes territoriaux: lecture d'une pratique habitante. Généalogie et description herméneutique des modalités de l'habiter en environnement urbain. - Berne: Peter Lang.

MuRPhy, R. (1993 [1987]): Vivre à corps perdu. - Paris: Presses Pocket.

Oliver, M. (1990): The politics of disablement. London: Macmillan.

Oliver, M. (1996): Understanding disability: from theory to practice. - London: Macmillan.

Organisation Mondiale de la Santé (1988): Classification internationale des handicaps. Déficiences, incapacités et désavantages. Un manuel de classification des conséquences des maladies. - Paris: CTNERHIINSERM.

Organisation Mondiale de la Santé (2001): Classification internationale du fonctionnement, du handicap et de la santé. - Genève: Organisation Mondiale de la Santé.

PARK, D.C., RAdFord, J.P. \& M.H. VICKeRs (1998): Disability studies in human geography. - In: Progress in Human Geography 22: 208-233.

PARR, H. (1997): Naming names: brief thoughts on disability and geography. - In: Area 29:173-176.

Philo, C. (2009): Disability. - In: Gregory, D., Johnston, R., Pratt, G., Watts, M.J. \& S. Whatmore (éds): The dictionary of human geography. $-5^{\text {th }}$ edition, Oxford: Blackwell: 164-165.

RAFFESTIN, C. (1980): Pour une géographie du pouvoir. - Paris: Librairies Techniques.

Ravaud, J.-F. (1999): Modèle individuel, modèle médical, modèle social: la question du sujet. - In: Handicap - Revue de Sciences Humaines et Sociales 81: 64-75.

RAvaUd, J.-F. (2001): Vers un modèle social du handicap. L'influence des organisations internationales et des mouvements des personnes handicapées. - In: DE RiEDMATTEN, R. (éd.): Une nouvelle approche de la dif- férence. Comment repenser le «handicap».- Genève: Médecine \& Hygiène: 55-68.

STIKER, H.-J. (2002): Analyse anthropologique comparée de deux classifications: Classification du fonctionnement et de la santé (OMS) et Classification québécoise: Processus de production du handicap (Société canadienne pour la CIDIH). - In: Handicap - Revue de Sciences Humaines et Sociales 94/95: 95-109.

Stiker, H.-J. (2005): Corps infirmes et sociétés. Essais d'anthropologie historique. - Paris: Dunod.

Turner, A. (1990 [1969]): Le phénomène rituel. Structure et contre-structure. - Paris: Presses Universitaires de France.

Van Gennep, A. (1981 [1909]): Les rites de passage. - Paris: Picard.

\section{Résumé: Les études du handicap en géographie. Fondements, état des lieux et ouvertures}

Alors que, historiquement, la géographie a négligé l'étude du handicap, un certain nombre de travaux, pour la plupart anglo-saxons, ont abordé la question ces dernières années, singulièrement selon les problématiques comportementales et historico-matérialistes. Ces travaux se sont fait l'écho des modèles issus des Disability Studies, opposant une explication individuelle à une explication sociale du handicap et, plus récemment, mobilisant une conception systémique. En revanche, les apports théoriques n'ont guère été intégrés. Cet article retrace ainsi les principaux modèles et théories issus des Disability Studies et l'évolution des études du handicap en géographie. Une attention particulière est portée aux théories des affordances et de la liminalité, en cela qu'elles semblent offrir un terreau fertile à l'investigation géographique des situations du handicap.

Mots-clés: géographie, théorie, handicap, affordance, liminalité

\section{Abstract: The study of disabilities in geography. Fun- damental concepts, current status and perspectives} Historically, geography has paid scant attention to disability. Research on the topic, most notably since the early 1990 s, has mostly stemmed from Anglophone geography and has followed behavioral and historical-materialist traditions. It has mirrored the disability models developed within Disability Studies, i.e. building on issues related to the individual or social models of disability, and, more recently, the ecological model. In contrast, theories of disability have hardly been considered within geographic research. This article presents the main models and theories of disability as well as the development of disability research within geography. Particular attention is paid to the theories of affordances and liminality, as both appear to provide promising avenues of research for a geography of disability. 
Keywords: geography, theory, handicap, affordance, liminality

Zusammenfassung: Handicap-Studien in der Geographie. Grundlagen, Bestandesaufnahme und Ausblick Historisch gesehen hat die Geographie Fragen der Behinderung wenig Beachtung geschenkt. In der letzten Zeit haben vor allem angelsächsische Arbeiten das Thema aufgegriffen, insbesondere mit Blick auf die Verhaltens- und historisch-materialistische Problematik. Diese Arbeiten widerspiegeln die von den Disability Studies entwickelten Ansätze, die individuelle und soziale Modelle der Behinderung einander gegenüberstellen und eine systemische Konzeption vorschlagen. Im Gegensatz dazu wurden Theorien der Behinderung in geographischen Untersuchungen bisher kaum behandelt. Dieser Artiekl zeigt sowohl die hauptsächlichen Modelle und Theorien der Behinderung auf, als auch die Entwicklung der Disability Studies innerhalb der Geographie. Besonderes Augenmerk wird der Affordance-Theorie und der Liminality-Theorie geschenkt, insofern als beide vielversprechende For- schungsmöglichkeiten für die geographische Untersuchung der Behinderung aufzeigen.

Schlüsselwörter: Geographie, Theorie, Handicap, affordance, liminality

Géogr. M.A. Jason Borioli, Institut de Géographie, Faculté des Géosciences et de l'Environnement, Université de Lausanne, Quartier Dorigny, Bâtiment Anthropole, CH-1015 Lausanne, et Laboratoire pour la Recherche Expérimentale du Comportement (LEEC), Institut de Psychologie, Faculté des Sciences Sociales et Politiques, Université de Lausanne, Suisse. e-mail: Jason.Borioli@unil.ch

\section{Manuskripteingang/received/manuscrit reçu le 30.7.2010}

Annahme zum Druck/accepted for publication/accepté pour publication: 9.12 .2010 